\title{
Evaluación del rendimiento diagnóstico de los criterios predictivos de la sociedad británica para el diagnóstico de coledocolitiasis en una población colombiana
}

\section{Diagnostic performance of the British Society of Gastroenterology predictive criteria for the diagnosis of choledocholithiasis in a Colombian population}

\author{
Johanna Gastelbondo-Morales, MD, ${ }^{1}$ (으 William Otero-Regino, MD, ${ }^{2 *}$ (1) Martín Gómez-Zuleta, MD. ${ }^{3}$ (1)
}

\author{
Gacceso abierto \\ Citación: \\ Gastelbondo-Morales J, Otero-Regino W, Gómez- \\ Zuleta M. Evaluación del rendimiento diagnóstico \\ de los criterios predictivos de la sociedad \\ británica para el diagnóstico de coledocolitiasis \\ en una población colombiana. Rev Colomb \\ Gastroenterol. 2020;35(3):269-279. https://doi. \\ org/10.22516/25007440.365 \\ Internista, Fellow de Gastroenterología; \\ Universidad Nacional de Colombia, Hospital \\ Universitario de Colombia. Bogotá D. C., \\ Colombia. \\ Profesor titular de Medicina, Unidad de \\ Gastroenterología; Universidad Nacional de \\ Colombia, Hospital Universitario Nacional \\ de Colombia, Gastroenterólogo en Clínica \\ Fundadores. Bogotá D. C., Colombia. \\ Profesor asociado de Medicina, Unidad de \\ Gastroenterología; Universidad Nacional de \\ Colombia, Hospital Universitario Nacional de \\ Colombia, Gastroenterólogo en Hospital de \\ Kennedy. Bogotá D. C., Colombia. \\ *Correspondencia: William Otero-Regino, MD \\ waoteror@gmail.com \\ Fecha recibido: $\quad 30 / 01 / 19$ \\ Fecha aceptado: $06 / 05 / 19$
}

\begin{abstract}
Resumen
Introducción: la coledocolitiasis (CLDL) puede ser difícil de diagnosticar. Su importancia radica en sus potenciales complicaciones y en que el tratamiento se realiza mediante colangiopancreatografía retrógrada endoscópica (CPRE), un procedimiento con riesgo de generar complicaciones. Se han propuesto guías para su diagnóstico y la más empleada es la de la ASGE (American Society for Gastrointestinal Endoscopy), cuyo rendimiento no es ideal. Recientemente, se ha publicado la guía británica. Este estudio se realizó para establecer el rendimiento de ambas guías. Materiales y métodos: estudio prospectivo realizado entre agosto 1 de 2017 y julio 31 de 2018. Resultados: se incluyeron 300 pacientes para el análisis. Se realizó una CPRE en 145 pacientes y se confirmó la existencia de CLDL en 124 de ellos (85,5 \%). La mediana de aspartato aminotransferasa (AST) y alanina aminotransferasa (ALT) fue mayor en los que tuvieron CLDC (207 mg/dL y $290 \mathrm{mg} / \mathrm{dl}$, respectivamente). Entre tanto, la tasa de complicaciones posteriores a la CPRE fue del 5,5\%. El análisis multivariado no encontró una asociación significativa para alguna variable predictora de CLDL. En pacientes con alta probabilidad, las guías británicas tuvieron una sensibilidad del $65 \%$ y una especificidad del $33 \%$, mientras que las guías ASGE mostraron una sensibilidad del $74 \%$ y una especificidad del $28 \%$. En probabilidad intermedia fueron menos eficientes. Conclusiones: los criterios de la ASGE y la BSG (British Society of Gastroenterology) no tienen un buen desempeño en la población estudiada, a fin de discriminar la existencia o no de CLDL. La guía de la ASGE mostró un mejor rendimiento en general que las guías británicas.
\end{abstract}

\section{Palabras claves}

Coledocolitiasis, rendimiento diagnóstico, predictores, guías, colangiopancreatografía retrógrada endoscópica

\begin{abstract}
Introduction: Choledocholithiasis (CDL) may be difficult to diagnose. The relevance of making a timely diagnosis lies in its potential negative effects and the fact that treatment requires performing endoscopic retrograde cholangiopancreatography (ERCP), which is a procedure with a high risk of complications. Several guidelines have been proposed for its diagnosis, including the ASGE Guidelines, which are the most widely used although they do not have an ideal performance, and the guidelines recently published by the BSG. The objective of this study was to compare the performance of both guidelines. Materials and methods: Prospective study carried out between August 1, 2017, and July 31,2018. Results: 300 patients were included for analysis. 145 underwent ERCP and choledocholithiasis was confirmed in 124 of them (85.5\%). Median AST and ALT levels were higher in patients with choledocholithiasis (207 mg/dL and $290 \mathrm{mg} / \mathrm{dL}$ ). The rate of post-ERCP complications was $5.5 \%$. Multivariate analysis found no significant association for any predictor of CDL. Regarding the "high probability" score, the BSG guidelines had sensitivity of $65 \%$ and specificity of $33 \%$, while the ASGE guidelines had sensitivity of $74 \%$ and specificity of $28 \%$. Both guidelines were less efficient for "intermediate probability". Conclusions: The ASGE and BSG criteria do not perform well in the population studied to determine whether they had CDL. The ASGE guidelines had a better overall performance than the BSG guidelines.
\end{abstract}

Keywords

Choledocholithiasis; Diagnostic performance; Predictors; Guidelines; ERCP. 


\section{INTRODUCCIÓN}

La coledocolitiasis (CLDL) es una enfermedad que afecta del 3 al $16 \%$ de los pacientes con cálculos biliares sintomáti$\cos (1)$. Cuando esta patología se presenta, puede producir complicaciones como pancreatitis aguda, ruptura del colédoco o colangitis (2). En la práctica, no es fácil diagnosticar la CLDL, ya que los diferentes exámenes de primera línea como la ecografía hepatobiliar o la tomografía axial computarizada (TAC) abdominal -solos o en combinación con pruebas bioquímicas- no tienen la exactitud deseada $(3,4)$.

En consecuencia, efectuar un diagnóstico correcto es fundamental porque la colangiopancreatografía retrógrada endoscópica (CPRE), que es el tratamiento de elección, puede tener complicaciones en el 5-10\% de los pacientes $(5,6)$. Por tanto, para minimizar la realización de CPRE innecesarias, expertos y sociedades científicas han propuesto diversos métodos, a fin de mejorar el diagnóstico de esa entidad $(7,8)$.

Entre estos, los más utilizados son los criterios de la Asociación Americana de Endoscopia (American Society for Gastrointestinal Endoscopy, ASGE) (7), los cuales clasifican la probabilidad de CLDL en tres grupos: probabilidad baja ( $<10 \%$ de presentar CLDL), probabilidad intermedia (10$50 \%)$ y probabilidad alta (>50 \%). Así pues, en los pacientes con probabilidad baja, se debe manejar la colelitiasis (colecistectomía) sin otros estudios para coledocolitiasis, mientras que en aquellos con probabilidad intermedia se recomienda realizar una evaluación adicional de la vía biliar (ecoendoscopia biliopancreática, resonancia magnética [RNM] o colangiografía intraoperatoria durante la colecistectomía laparoscópica, si hay indicación de esta cirugía). Entre tanto, quienes tienen una probabilidad alta requieren ser conducidos a una CPRE.

No obstante, cuando tales criterios se aplican en algunas poblaciones, el diagnóstico definitivo de CLDL en aquellos con probabilidad alta se logra solo en el $60 \%$ de los pacientes. Esto refleja que en el $40 \%$ de ellos se han realizado CPRE innecesarias $(9,10)$. Recientemente, la Sociedad Británica de Gastroenterología (British Society of Gastroenterology, BSG) (8) diseñó una nueva escala con el fin de mejorar el rendimiento. Sin embargo, hasta el momento no existen estudios que hayan investigado su verdadero rendimiento. La diferencia fundamental entre estas dos guías, la ASGE y la BSG, es que la británica asigna puntos a los hallazgos ecográficos y al perfil hepático, según sus alteraciones.

En Colombia no se han realizado investigaciones con estas escalas y solo existen algunas indagaciones sobre la CLDL, pero con otros propósitos (11-13). Dentro de este contexto, en el trabajo de Vargas y colaboradores (11) se estudió la concordancia entre la colangiografía por RNM y la CPRE, mientras que otros estudios han evaluado factores predictivos aislados para la $\operatorname{CLDL}(12,13)$.

Por otro lado, el estudio de Gómez y colaboradores (12) encontró que, en pacientes con colelitiasis, la presencia de una bilirrubina directa $>30 \%$ del total tiene un riesgo de 9,7 veces para CLDL y de 4,3 veces para un hallazgo positivo en CPRE (coledocolitiasis o una vía biliar dilatada). Si esa alteración ocurre en pacientes mayores de 55 años, el riesgo para una CLDL es de 11,3.

En otro estudio, también realizado de forma reciente en la Universidad Nacional, Yurgaky y colaboradores (13) encontraron que los pacientes con cólico biliar y una elevación inicial de ALT y AST -con descenso de sus valores a las 48 y $72 \mathrm{~h}$ - tienen un riesgo de 4,2 (intervalo de confianza [IC], $95 \%: 1,98-9.02$ ) para CLDL.

Asimismo, al revisar la literatura de América Latina, incluida la de Colombia, no se encuentran estudios que hayan validado las recientes guías británicas (8), a fin de predecir la CLDL. En consecuencia, se decidió realizar la presente investigación para evaluar los criterios diagnósticos de las guías británicas para la CLDL y comparar su rendimiento frente a los de la ASGE.

\section{MATERIALES Y MÉTODOS}

Estudio prospectivo realizado en el Hospital Universitario Nacional de Colombia entre agosto 01 de 2017 y el 31 de julio de 2018. Se incluyeron pacientes hospitalizados, mayores de 18 años, con sospecha clínica o paraclínica de CLDL. La verificación de la CLDL se hizo con CPRE o con colangiografía por resonancia magnética (CRM). En efecto, la CPRE fue el método de referencia. Los criterios para las diferentes probabilidades se basaron en las guías de la ASGE (7), así como en las guías británicas (8).

En estas guías, la probabilidad de CLDL puede ser baja, intermedia y alta. Así pues, en la probabilidad baja todo se observa normal para ambas guías, mientras que en la probabilidad intermedia (británicas) se encuentra una dilatación de la vía biliar común (VBC) durante la ecografía, y las pruebas hepáticas normales o las pruebas hepáticas alteradas presentan una VBC normal. En la probabilidad alta (británicas) ocurre la identificación de cálculos en la VBC, mediante una ecografía hepatobiliar, así como de colangitis, cólico biliar con ictericia y dilatación de la VBC.

Las guías de la ASGE (7) estratifican las probabilidad según predictores, los cuales se clasifican en tres categorías: muy fuertes (evidencia de cálculo en ecografía, colangitis ascendente clínica, o bilirrubina total $\geq 4 \mathrm{mg} / \mathrm{dL}$ ), fuertes (conducto común biliar dilatado $>6 \mathrm{~mm}$, bilirrubina total entre $1,8-4 \mathrm{mg} / \mathrm{dL}$ ) y moderados (perfil hepático alterado diferente a las bilirrubinas, edad mayor a 55 años o pancreatitis biliar). De acuerdo con estos predictores se definen criterios como la 
probabilidad intermedia (presencia de un predictor fuerte o de alguno moderado) y la probabilidad alta (presencia de los dos predictores fuertes o cualquiera muy fuerte).

\section{Criterios de exclusión}

Se excluyeron pacientes con historia de colecistectomía (por la alteración que se puede presentar en el diámetro del colédoco); lesiones biliares conocidas o encontradas actualmente; enfermedad hepática crónica por historia clínica o por perfil hepático alterado de base, y personas que hubieran tenido una manipulación previa de la vía biliar. El protocolo de investigación y el consentimiento informado fueron aprobados por los comités de Investigación y Ética de la Facultad de Medicina de la Universidad Nacional de Colombia y del Hospital Universitario Nacional de Colombia. Todos los pacientes firmaron el consentimiento informado.

\section{Análisis estadístico}

La investigación se planteó metodológicamente como una evaluación del rendimiento de una herramienta diagnóstica, por lo que no se requirió calcular un número específico de pacientes para constituir la muestra. Por lo tanto, se recolectaron todos los pacientes que cumplieran los criterios de inclusión y exclusión, atendidos durante el período de estudio. Este tipo de muestras se conoce como muestreo por conveniencia (no probabilístico). El análisis estadístico se realizó con Stata v.15. Las variables cuantitativas que tuvieron una distribución normal se presentaron como medias con desviación estándar (DE), mientras que aquellas que no tuvieron distribución normal como medianas con rangos intercuartílicos, y las variables categóricas como frecuencias absolutas y relativas.

Para cada categoría de riesgo, predictor y combinación de predictores se utilizó la prueba Chi-cuadrado $\left(\chi^{2}\right)$, a fin de evaluar la asociación con la presencia de CLDL. Además, se realizó un análisis de regresión logística bivariado y multivariado para calcular el Odds Ratio (OR) entre cada predictor y la presencia de CLDL. Se determinó la sensibilidad, la especificidad, el valor predictivo positivo (VPP) y el valor predictivo negativo (VPN), así como el cociente de verosimilitudes (Likelihood Ratio, $L R$ ) positivo $(\mathrm{LR}+$ ) y negativo (LR -) de cada algoritmo a estudio para la presencia de CLDL. Las características operativas se calcularon utilizando la CPRE como el método de referencia (gold standard), con un nivel de significancia del $5 \%$.

\section{RESULTADOS}

Durante el período de estudio, fueron elegibles 521 pacientes con sospecha de CLDL. Luego de ello, se incluyeron
300 pacientes, pero se excluyeron 229 por diversas causas (Figura 1). La edad promedio fue de 52 años. El $59 \%$ (177 pacientes) fueron mujeres. El tiempo promedio de evolución de los síntomas fue de 6 días. En la Tabla 1 se muestran las características generales, los hallazgos ultrasonográficos y por CRM, así como las sospechas diagnósticas adicionales como colangitis y pancreatitis biliar entre los pacientes con y sin CLDL. La CLDL fue confirmada por CPRE en 124 pacientes.

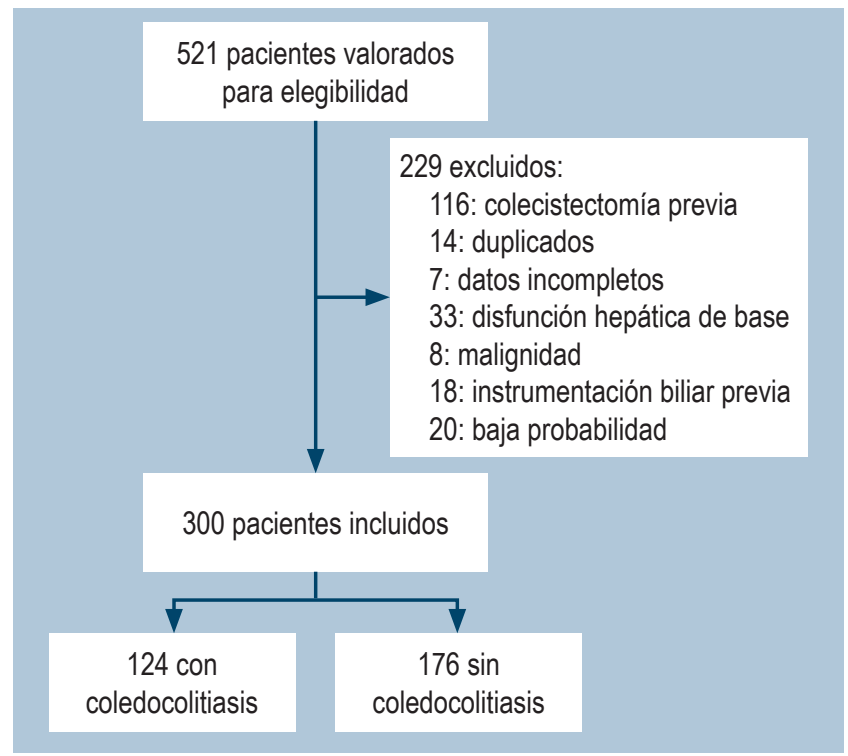

Figura 1. Pacientes elegibles e incluidos en el estudio.

\section{Pacientes con pancreatitis biliar}

De los 83 pacientes con pancreatitis biliar, a $71(85,5 \%)$ se les realizó una CRM y se observaron cálculos en la vía biliar en 14 pacientes (19,7 \%). La CPRE confirmó la CLDL en todos los pacientes. Asimismo, a 4 pacientes, en quienes la CRM no mostró CLDL pero tenían alta probabilidad de ella, se les realizó CPRE. Esta encontró CLDL en 2 de ellos ( $50 \%)$. En contraste, en 12 pacientes con alta probabilidad de CLDL no se realizó una RNM, pero la CPRE encontró CLDL en 10 casos ( $83 \%)$.

\section{Pacientes llevados a CPRE}

Un total de 145 pacientes fueron llevados a CPRE, cuyas características y hallazgos imagenológicos se describen en la Tabla 2. Los pacientes que no presentaron CLDL tenían mayor tiempo de evolución de los síntomas, en comparación con los que sí tuvieron CLDL ( 12 frente a 7,5 días). Los valores del perfil hepático están reportados en medianas y rangos intercuartílicos, ya que no tuvieron una distribución normal. Se encontraron valores extremos en cada uno de ellos, lo que 
Tabla 1. Características basales de los pacientes con sospecha de coledocolitiasis

\begin{tabular}{|c|c|c|c|c|c|c|}
\hline \multirow[t]{2}{*}{ Características clínicas } & \multicolumn{2}{|c|}{ Total $(n=300)$} & \multicolumn{2}{|c|}{ Sí CLDL (n = 124) } & \multicolumn{2}{|c|}{ No CLDL $(n=176)$} \\
\hline & $\mathbf{n}$ & $\%$ & $n$ & $\%$ & $n$ & $\%$ \\
\hline Edad-promedio (DE) & 52 & $(18,6)$ & 55,5 & $(20,09)$ & 51 & $(17,4)$ \\
\hline \multicolumn{7}{|l|}{ Edad: mayor a 55 años } \\
\hline Sí (\%) & 140 & $(46,7)$ & 61 & $(20,3)$ & 79 & $(26,3)$ \\
\hline No (\%) & 160 & $(53,3)$ & 63 & $(21,1)$ & 97 & $(32,3)$ \\
\hline \multicolumn{7}{|l|}{ Sexo } \\
\hline Femenino (\%) & 177 & (59) & 72 & (24) & 105 & (35) \\
\hline Masculino (\%) & 123 & $(41)$ & 52 & $(17,33)$ & 71 & $(23,66)$ \\
\hline Tiempo de evolución de síntomas promedio (DE) & 6,11 & $(12)$ & 7,3 & $(13,3)$ & 5,1 & $(10,94)$ \\
\hline \multicolumn{7}{|l|}{ Laboratorio } \\
\hline BT promedio (DE) & 4 & $(12)$ & 5,8 & $(18,6)$ & 2,72 & $(2,78)$ \\
\hline \multicolumn{7}{|l|}{$\mathrm{BT} \geq 4$} \\
\hline Sí (\%) & 95 & $(31,67)$ & 57 & $(19)$ & 38 & $(12,66)$ \\
\hline No $(\%)$ & 205 & $(68,33)$ & 67 & $(22,33)$ & 138 & $(46)$ \\
\hline AST promedio (DE) & 280,22 & $(346,98)$ & 268,40 & $(251,1)$ & 288,33 & $(400,15)$ \\
\hline ALT promedio (DE) & 328,93 & $(302,66)$ & 356,37 & $(282,05)$ & 310,26 & $(315,34)$ \\
\hline Fosfatasa alcalina (DE) & 244,4 & $(187,4)$ & 281 & $(182,8)$ & 218,79 & $(186,8)$ \\
\hline \multicolumn{7}{|l|}{ Radiológicas } \\
\hline \multicolumn{7}{|l|}{ Cálculo en colédoco ultrasonido } \\
\hline Sí (\%) & 31 & $(10,47)$ & 26 & $(8,66)$ & 5 & $(1,66)$ \\
\hline No $(\%)$ & 269 & $(89,19)$ & 98 & $(32)$ & 171 & $(63,66)$ \\
\hline \multicolumn{7}{|l|}{ Cálculo en colédoco CRM ( $n=236)$} \\
\hline Sí (\%) & 63 & $(26,69)$ & 61 & $(20,33)$ & 2 & $(0,66)$ \\
\hline No $(\%)$ & 173 & $(73,31)$ & 11 & $(3,66)$ & 162 & (54) \\
\hline \multicolumn{7}{|l|}{ Sospechas diagnósticas asociadas } \\
\hline \multicolumn{7}{|l|}{ Colangitis biliar ascendente } \\
\hline Sí (\%) & 30 & $(10,03)$ & 22 & $(7,33)$ & 8 & $(2,66)$ \\
\hline No $(\%)$ & 269 & $(89,97)$ & 101 & $(33,66)$ & 168 & (56) \\
\hline \multicolumn{7}{|l|}{ Pancreatitis biliar } \\
\hline Sí (\%) & 83 & $(27,85)$ & 26 & $(8,66)$ & 57 & (19) \\
\hline No $(\%)$ & 215 & $(72,15)$ & 98 & $(32,66)$ & 117 & (39) \\
\hline
\end{tabular}

CLDL: coledocolitiasis; BT: bilirrubina total; DE: desviación estándar; AST: aspartato aminotransferasa; ALT: alanina aminotransferasa; CRM: colangiografía por resonancia magnética. 
Tabla 2. Características de base de los pacientes llevados a CPRE

\begin{tabular}{|c|c|c|c|c|c|c|}
\hline \multirow[t]{2}{*}{ Características clínicas } & \multicolumn{2}{|c|}{ Total $(n=145)$} & \multicolumn{2}{|c|}{ Coledocolitiasis (124) } & \multicolumn{2}{|c|}{ No coledocolitiasis (21) } \\
\hline & $\mathbf{n}$ & $\%$ & $\mathbf{n}$ & $\%$ & $\mathbf{n}$ & $\%$ \\
\hline Edad-promedio (DE) & 55,11 & $(19,5)$ & 55,58 & $(20,09)$ & 52,33 & $(16,52)$ \\
\hline \multicolumn{7}{|l|}{ Edad: mayor a 55 años } \\
\hline Sí (\%) & 72 & $(49,66)$ & 61 & $(49,20)$ & 10 & $(47,61)$ \\
\hline No $(\%)$ & 73 & $(50,34)$ & 63 & $(50,80)$ & 11 & $(52,39)$ \\
\hline \multicolumn{7}{|l|}{ Sexo } \\
\hline Femenino (\%) & 83 & $(57,24)$ & 72 & $(58,06)$ & 11 & $(52,39)$ \\
\hline Tiempo de evolución de síntomas - promedio (DE) & 8,2 & $(14,88)$ & 7,53 & $(13,31)$ & 12,47 & $(21,97)$ \\
\hline \multicolumn{7}{|l|}{ Laboratorio } \\
\hline BT promedio (DE) & 5,9 & $(17,3)$ & 5,84 & $(18,64)$ & 6,26 & $(4,18)$ \\
\hline \multicolumn{7}{|l|}{$\mathrm{BT} \geq 4$} \\
\hline Sí $(\%)$ & 70 & $(48,28)$ & 57 & $(45,96)$ & 13 & $(61,9)$ \\
\hline No $(\%)$ & 75 & $(51,72)$ & 67 & $(54,04)$ & 8 & $(38,1)$ \\
\hline AST mediana (RIQ) & 201 & $(99-363)$ & 207 & $(100-362,5)$ & 139 & $(93-443)$ \\
\hline ALT mediana (RIQ) & 289.5 & $119-518,5$ & 290 & $(116-513)$ & 263 & $(127-529)$ \\
\hline Fosfatasa alcalina mediana (RIQ) & 227 & $158-396$ & 222,5 & $(153-355)$ & 308 & $205-308$ \\
\hline \multicolumn{7}{|l|}{ Cálculo en colédoco ultrasonido } \\
\hline Sí & 29 & $(20,14)$ & 26 & $(20,96)$ & 3 & $(14,28)$ \\
\hline No & 116 & $(79,86)$ & 98 & $(79,04)$ & 18 & $(85,72)$ \\
\hline \multicolumn{7}{|l|}{ Subgrupos (n diferente) } \\
\hline Cálculo en colédoco, colangiografía por resonancia magnética & $\mathrm{n}=82$ & & $n=72$ & & $n=10$ & \\
\hline Sí (\%) & 61 & (74) & 60 & (98) & 1 & $(1,6)$ \\
\hline No $(\%)$ & 20 & (24) & 12 & $(60)$ & 9 & $(45)$ \\
\hline \multicolumn{7}{|l|}{ Sospechas diagnósticas adicionales } \\
\hline \multicolumn{7}{|l|}{ Colangitis biliar ascendente } \\
\hline Sí (\%) & 29 & $(19,58)$ & 23 & $(79,31)$ & 6 & $(20,68)$ \\
\hline \multicolumn{7}{|l|}{ Pancreatitis biliar } \\
\hline Sí (\%) & 30 & $(20,83)$ & 26 & (86) & 4 & $(13,3)$ \\
\hline
\end{tabular}

CPRE: colangiopancreatografía retrógrada endoscópica; BT: bilirrubina total; DE: desviación estándar; AST: aspartato aminotransferasa; ALT: alanina aminotransferasa; RIQ: rango intercuartílico (Interquartile Range).

resultó en una mediana de AST y ALT mayor en los pacientes con CLDL, en comparación con los que no la tuvieron ( 207 y 290 frente 139 y 263 , respectivamente).
De igual manera, en 29 pacientes (20\%) se evidenció un cálculo en la vía biliar por ecografía, el cual fue corroborado en la CPRE en el $89 \%$ de dichos pacientes. Entre 
tanto, la CRM fue realizada en 82 pacientes, de los cuales el $74 \%$ mostró cálculos en la vía biliar. Esto fue confirmado con la CPRE en el 98 \% de los casos. En contraste, cuando la CRM no evidenció cálculos en la vía biliar ( $24 \%)$, se encontraron cálculos por CPRE en el $60 \%$ de los enfermos. La pancreatitis biliar se presentó en el $20 \%$ de los pacientes y el $86 \%$ de ellos presentaron CLDL.

Los principales hallazgos de las CPRE se muestran en la Figura 2.

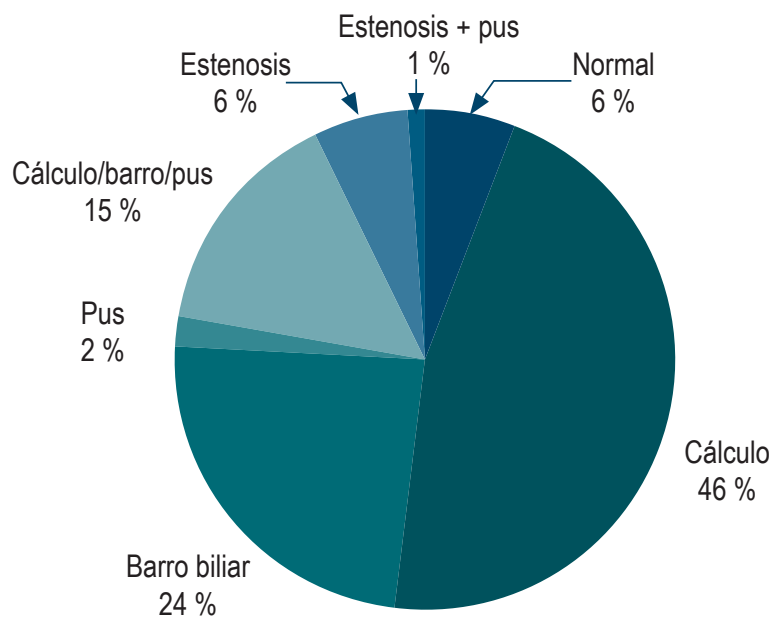

Figura 2. Hallazgos de la CPRE. CPRE: colangiopancreatografía retrógrada endoscópica

La tasa de complicaciones fue del 5,5\%. La pancreatitis posterior a la CPRE fuela más frecuentey se dio en un $4,13 \%$, seguida por el sangrado papilar, que ocurrió en un 1,37 \% de los pacientes llevados a CPRE.

\section{Criterios diagnósticos ASGE}

Del total de pacientes, 37 cumplieron con los criterios de probabilidad intermedia, mientras que 107 los de probabilidad alta. Entre tanto, solo 1 paciente cumplió los criterios de probabilidad baja e ingreso, ya que, según las guías de la BSG, era de probabilidad intermedia. Cuando los pacientes tuvieron probabilidad intermedia y alta, se encontraron cálculos biliares en el 83 y el $85 \%$ de estos, respectivamente. La correlación con la presencia o no de la CLDL se muestra en la Tabla 3.

En el análisis bivariado y multivariado, no hubo diferencia estadísticamente significativa con la presencia de CLDL (Tablas 4 y 5 ).

\section{Criterios diagnósticos BSG}

Un total de 50 pacientes cumplieron con los criterios de probabilidad intermedia y 95 de probabilidad alta. En esos dos grupos, se encontró CLDL en 86 y 85 \% de los pacientes, respectivamente. La correlación con la presencia o no de CLDL se muestra en la Tabla 6.

Tabla 3. Presencia o no de coledocolitiasis según la categoría de riesgo de las guías ASGE

\begin{tabular}{|ccccccc|} 
Características & Total & \multicolumn{2}{c}{ Coledocolitiasis } & \multicolumn{2}{c|}{$\begin{array}{c}\text { No } \\
\text { coledocolitiasis }\end{array}$} \\
\hline Probabilidad & $\mathrm{n}$ & $\%$ & $\mathrm{n}$ & $\%$ & $\mathrm{n}$ & $\%$ \\
\hline Baja & 1 & 0,68 & 1 & $(100)$ & 0 & $(0)$ \\
\hline Intermedia & 37 & 25,51 & 31 & $(83,78)$ & 6 & $(16,22)$ \\
\hline Alta & 107 & 7379 & 9231 & $(85,84)$ & 15 & $(14,16)$ \\
\hline
\end{tabular}

Tabla 4. Análisis bivariado. Asociación con CLDL, guías ASGE

\begin{tabular}{|c|c|c|c|}
\hline & OR & IC, $95 \%$ & $p$ \\
\hline \multicolumn{4}{|l|}{ Predictores muy fuertes } \\
\hline Bilirrubina $>4$ mg/dL & 0,52 & $0,20-1,35$ & 0,17 \\
\hline Cálculos en el colédoco US & 1,65 & $0,45-6,13$ & 0,31 \\
\hline Sospecha de coledocolitiasis & 0,54 & $0,18-1,56$ & 0,27 \\
\hline \multicolumn{4}{|l|}{ Predictores muy fuertes } \\
\hline Bilirrubina 1,8-4 mg/dL & 1,07 & $0,40-2,86$ & 0,88 \\
\hline Dilatación en US & 1,01 & $0,36-2,82$ & 0,98 \\
\hline \multicolumn{4}{|l|}{ Moderados } \\
\hline Pancreatitis & 1,13 & $0,35-3,67$ & 0,82 \\
\hline Edad $>55$ años & 0,88 & $0,34-2,22$ & 0,78 \\
\hline
\end{tabular}

CLDL: coledocolitiasis; ASGE: Asociación Americana de Endoscopia (American Society for Gastrointestinal Endoscopy); OR: Odds Ratio; IC: intervalo de confianza; US: ultrasonido.

Tabla 5. Análisis multivariado. Asociación con CLDL, guías ASGE

\begin{tabular}{|c|c|c|c|}
\hline & OR & IC, $95 \%$ & $p$ \\
\hline \multicolumn{4}{|l|}{ Predictores muy fuertes } \\
\hline Cálculo en UST & 0,60 & $0,16-2,26$ & 0,45 \\
\hline Bilirrubina $>4 \mathrm{mg} / \mathrm{dL}$ & 1,54 & $0,56-4,22$ & 0,39 \\
\hline Colangitis & 1,58 & $0,52-4,83$ & 0,41 \\
\hline \multicolumn{4}{|l|}{ Predictores fuertes } \\
\hline Dilatación en US & 0,98 & $0,35-2,75$ & 0,97 \\
\hline Bilirrubina $1,8-4 \mathrm{mg} / \mathrm{dL}$ & 0,96 & & 0,93 \\
\hline \multicolumn{4}{|l|}{ Moderados } \\
\hline Pancreatitis & 1,13 & $0,44-2,86$ & 0,79 \\
\hline Edad >55 años & 0,89 & $0,27-2,87$ & 0,84 \\
\hline
\end{tabular}

CLDL: coledocolitiasis; ASGE: Asociación Americana de Endoscopia (American Society for Gastrointestinal Endoscopy); OR: Odds Ratio; IC: intervalo de confianza; US: ultrasonido; UST: ultrasonido terapéutico. 
Tabla 6. Presencia de CLDL según la categoría de riesgo de las guías BSG

\begin{tabular}{|lcccccc|} 
& \multicolumn{2}{c}{ Total } & \multicolumn{2}{c|}{ Coledocolitiasis } & \multicolumn{2}{c|}{$\begin{array}{c}\text { No } \\
\text { coledocolitiasis }\end{array}$} \\
\hline Probabilidad & $\mathrm{n}$ & $\%$ & $\mathrm{n}$ & $\%$ & $\mathrm{n}$ & $\%$ \\
\hline Intermedia & 50 & 33,79 & 43 & $(86)$ & 7 & $(14)$ \\
Alta & 95 & 65,51 & 81 & $(85)$ & 14 & $(15)$ \\
\hline
\end{tabular}

CLDL: coledocolitiasis; BSG: Sociedad Británica de Gastroenterología (British Society of Gastroenterology).

En el análisis bivariado y multivariado, no hubo una diferencia estadísticamente significativa entre la presencia $o$ no de CLDL. Los resultados se presentan a continuación (Tablas 7 y 8 ).

Tabla 7. Análisis bivariado. Asociación con CLDL, guías BSG

\begin{tabular}{|c|c|c|c|}
\hline & OR & IC, $95 \%$ & $p$ \\
\hline \multicolumn{4}{|l|}{ Predictores de probabilidad intermedia } \\
\hline Dilatación >6 mm & 1,01 & $0,36-2,82$ & 0,98 \\
\hline $\begin{array}{l}\text { Alguna de las pruebas de función } \\
\text { hepática normal }\end{array}$ & 1,70 & $0,64-4,50$ & 0,27 \\
\hline \multicolumn{4}{|l|}{ Predictores de probabilidad intermedia } \\
\hline Dilatación $<6$ mm & 0,98 & $0,35-2,75$ & 0,98 \\
\hline $\begin{array}{l}\text { Alguna de las pruebas de función } \\
\text { hepática elevada }\end{array}$ & 0,83 & $0,97-7,16$ & 0,86 \\
\hline \multicolumn{4}{|l|}{ Predictores de probabilidad alta } \\
\hline Cálculo en US & 1,65 & $0,45-6,13$ & 0,31 \\
\hline Sospecha de colangitis & 0,54 & $0,18-1,56$ & 0,27 \\
\hline $\begin{array}{l}\text { Dilatación de colédoco y bilirrubinas } \\
\text { elevadas }\end{array}$ & 0,56 & $0,21-1,50$ & 0,24 \\
\hline
\end{tabular}

CLDL: coledocolitiasis; BSG: Sociedad Británica de Gastroenterología (British Society of Gastroenterology); OR: Odds Ratio; IC: intervalo de confianza.

\section{Características de rendimiento comparativo entre guías}

Al comparar la proporción y el rendimiento de los dos algoritmos, se encontró que, en los pacientes con probabilidad alta, la sensibilidad para las guías de la BSG y la ASGE fue del 65 y el $74 \%$, respectivamente. En cambio, la especificidad fue ligeramente superior para la BSG en comparación con la ASGE (33\% frente al $28 \%$ ). El VPP fue excelente y similar en ambas guías (BSG: $85 \%$; ASGE: $86 \%$ ). En contraste, el VPN fue muy bajo y similar en ambas guías y la exactitud fue de mayor para las de ASGE, en comparación con el $39 \%$ para las británicas (Tabla 9).

Tabla 8. Análisis multivariado. Asociación con CLDL, guías BSG

\begin{tabular}{|c|c|c|c|}
\hline & OR & IC, $95 \%$ & $p$ \\
\hline \multicolumn{4}{|l|}{ Predictores de probabilidad intermedia } \\
\hline Dilatación >6 mm & 1,16 & $0,37-3,59$ & 0,79 \\
\hline $\begin{array}{l}\text { Alguna de las pruebas de función } \\
\text { hepática normal }\end{array}$ & 0,26 & $0,23-2,94$ & 0,27 \\
\hline \multicolumn{4}{|l|}{ Predictores de probabilidad intermedia } \\
\hline Dilatación $<6$ mm & 0,98 & $0,35-2,95$ & 0,97 \\
\hline $\begin{array}{l}\text { Alguna de las pruebas de función } \\
\text { hepática elevada }\end{array}$ & 1,14 & $0,27-2,23$ & 0,2 \\
\hline \multicolumn{4}{|l|}{ Predictores de probabilidad alta } \\
\hline Cálculo en US & 0,50 & $0,13-1,93$ & 0,32 \\
\hline Sospecha de colangitis & 1,91 & $0,65-5,60$ & 0,23 \\
\hline $\begin{array}{l}\text { Dilatación de colédoco y bilirrubinas } \\
\text { elevadas }\end{array}$ & 1,79 & $0,66-4,80$ & 0,24 \\
\hline
\end{tabular}

CLDL: coledocolitiasis; BSG: Sociedad Británica de Gastroenterología (British Society of Gastroenterology); OR: Odds Ratio; IC: intervalo de confianza.

Tabla 9. Pacientes con alta probabilidad para coledocolitiasis y sus características de rendimiento

\begin{tabular}{|lcc|}
\hline & $\begin{array}{c}\text { Algoritmo BSG } \\
(\mathbf{n}=\mathbf{1 4 5})\end{array}$ & $\begin{array}{c}\text { Algoritmo ASGE } \\
(\mathbf{n}=\mathbf{1 4 5})\end{array}$ \\
\hline $\begin{array}{l}\text { Pacientes categorizados como } \\
\text { alto riesgo (\%) }\end{array}$ & $95(65)$ & $107(73)$ \\
\hline Sensibilidad & 0,65 & 0,74 \\
\hline Especificidad & 0,33 & 0,28 \\
\hline Valor predictivo positivo & 0,85 & 0,86 \\
\hline Valor predictivo negativo & 0,14 & 0,15 \\
\hline Exactitud & 0,39 & 0,67 \\
\hline LR positivo & 0,97 & 0,90 \\
\hline LR negativo & 1,04 & 1,03
\end{tabular}

LR: cociente de verosimilitudes (Likelihood Ratio); BSG: Sociedad Británica de Gastroenterología (British Society of Gastroenterology); ASGE: Asociación Americana de Endoscopia (American Society for Gastrointestinal Endoscopy).

El rendimiento de las guías BSG y ASGE es malo para los casos con probabilidad intermedia, pues se observa una baja sensibilidad ( 34 y $35 \%$, respectivamente) y una especificidad moderada (66 y $71 \%$, respectivamente); la 
exactitud de las británicas fue mayor que la de ASGE: $60 \%$ frente al $32 \%$ (Tabla 10).

Tabla 10. Pacientes con probabilidad intermedia para coledocolitiasis y sus características de rendimiento

\begin{tabular}{lcc|}
\hline & $\begin{array}{c}\text { Algoritmo BSG } \\
(\mathbf{n}=\mathbf{1 4 5})\end{array}$ & $\begin{array}{c}\text { Algoritmo ASGE } \\
(\mathbf{n}=\mathbf{1 4 5})\end{array}$ \\
\hline $\begin{array}{l}\text { Pacientes categorizados como } \\
\text { riesgo intermedio (\%) }\end{array}$ & $50(34)$ & $37(25)$ \\
\hline Sensibilidad & 0,34 & 0,25 \\
\hline Especificidad & 0,66 & 0,71 \\
\hline Valor predictivo positivo & 0,86 & 0,84 \\
\hline Valor predictivo negativo & 0,14 & 0,14 \\
\hline Exactitud & 0,60 & 0,32 \\
\hline LR positivo & 1,04 & 0,90 \\
\hline LR negativo & 0,97 & 1,03
\end{tabular}

LR: cociente de verosimilitudes (Likelihood Ratio); BSG: Sociedad Británica de Gastroenterología (British Society of Gastroenterology); ASGE: Asociación Americana de Endoscopia (American Society for Gastrointestinal Endoscopy).
Al comparar el rendimiento diagnóstico de las guías de la ASGE con lo descrito en la literatura se encuentran aspectos que se detallan en la Tabla 11.

\section{DISCUSIÓN}

En el presente estudio, se encontró CLDL en 124 pacientes $(85 \%)$ de los que fueron conducidos a CPRE. Si se analiza este hallazgo, puede significar que solo el $15 \%$ de las CPRE realizadas fueron innecesarias o negativas. En diversos estudios internacionales, el rendimiento reportado de este procedimiento es inferior $(14,15,17,18)$. En un estudio, las CPRE negativas ocurrieron en el $44 \%$ de los pacientes (17), y más recientemente, en un trabajo de Benites y colaboradores (18), y en otro de Rubin y colaboradores (14), las tasas de CPRE negativas fueron del 25 y $34 \%$, respectivamente.

En efecto, el de Rubin (14) es un estudio retrospectivo que utilizó las guías ASGE. En otros estudios, como el de Magalhães (15) y el de Adams (16), las CPRE normales se encontraron en el 28 y el $25 \%$ de los pacientes, respectivamente. Sin embargo, es importante aclarar que en el estudio de Adams (16), la CRM y la ecoendoscopia biliopancreática también se utilizaron como métodos de confirmación de CLDL, además de la CPRE. Por tanto, no todos los

Tabla 11. Evaluación del rendimiento criterios ASGE en otros estudios

\begin{tabular}{|c|c|c|c|c|c|c|c|c|}
\hline & & $\mathbf{n}$ & & DL & & CLDL & Sensibilidad & Especificidad \\
\hline Rubin (Estados Unidos-2013) (14) & $\begin{array}{l}\text { PA } \\
\text { PI }\end{array}$ & $\begin{array}{l}249 \\
193\end{array}$ & $\begin{array}{l}189 \\
102\end{array}$ & $\begin{array}{l}71,59 \% \\
40,96 \%\end{array}$ & $\begin{array}{c}75 \\
147\end{array}$ & $\begin{array}{c}28,41 \% \\
59 \%\end{array}$ & NR & NR \\
\hline Magalhaes (Portugal-2015) (15) & $\begin{array}{l}\text { PA } \\
\text { PI }\end{array}$ & $\begin{array}{c}73 \\
179\end{array}$ & $\begin{array}{l}154 \\
25\end{array}$ & $\begin{array}{l}79,70 \% \\
34,25 \%\end{array}$ & $\begin{array}{l}39 \\
48\end{array}$ & $\begin{array}{l}20,21 \% \\
65,75 \%\end{array}$ & $86 \%$ & $56,20 \%$ \\
\hline Adams (Estados Unidos-2015) (16) & $\begin{array}{l}\text { PA } \\
\text { NPA }\end{array}$ & $\begin{array}{l}319 \\
319\end{array}$ & $\begin{array}{c}99 \\
111\end{array}$ & $\begin{array}{l}55,31 \% \\
34,80 \%\end{array}$ & $\begin{array}{c}80 \\
208\end{array}$ & $\begin{array}{l}44,69 \% \\
65,20 \%\end{array}$ & $\begin{array}{l}47 \% \\
46 \%\end{array}$ & $\begin{array}{l}73 \% \\
76 \%\end{array}$ \\
\hline Narváez-Rivera (México- 2016) (17) & $\begin{array}{l}\text { PA } \\
\text { PI }\end{array}$ & $\begin{array}{c}208 \\
48\end{array}$ & $\begin{array}{c}124 \\
21\end{array}$ & $\begin{array}{c}59 \% \\
43,70 \%\end{array}$ & $\begin{array}{l}84 \\
27\end{array}$ & $\begin{array}{c}40,30 \% \\
56 \%\end{array}$ & $\begin{array}{l}85,50 \% \\
14,40 \%\end{array}$ & $\begin{array}{l}24,30 \% \\
75,60 \%\end{array}$ \\
\hline Sethi (China-2016) (10) & $\begin{array}{l}\text { PA } \\
\text { PI }\end{array}$ & $\begin{array}{c}244 \\
92\end{array}$ & $\begin{array}{c}185 \\
45\end{array}$ & $\begin{array}{l}75,80 \% \\
48,90 \%\end{array}$ & $\begin{array}{l}59 \\
47\end{array}$ & $\begin{array}{c}24,10 \% \\
51 \%\end{array}$ & $\begin{array}{l}80,40 \% \\
19,57 \%\end{array}$ & $\begin{array}{l}44,34 \% \\
55,66 \%\end{array}$ \\
\hline Benites (Perú-2017) (18) & $\begin{array}{l}\text { PA } \\
\text { PI }\end{array}$ & $\begin{array}{l}91 \\
27\end{array}$ & $\begin{array}{l}69 \\
19\end{array}$ & $\begin{array}{l}75,82 \% \\
70,37 \%\end{array}$ & $\begin{array}{c}22 \\
8\end{array}$ & $\begin{array}{l}24,18 \% \\
29,63 \%\end{array}$ & $\begin{array}{c}78,40 \% \\
76 \%\end{array}$ & $\begin{array}{c}26,70 \% \\
63 \%\end{array}$ \\
\hline Ebrahim (Dinamarca-2018) (19) & $\begin{array}{l}\text { PA } \\
\text { PI }\end{array}$ & $\begin{array}{l}124 \\
181\end{array}$ & $\begin{array}{l}\text { NR } \\
\text { NR }\end{array}$ & $\begin{array}{l}\text { NR } \\
\text { NR }\end{array}$ & $\begin{array}{l}\text { NR } \\
\text { NR }\end{array}$ & $\begin{array}{l}\text { NR } \\
\text { NR }\end{array}$ & $\begin{array}{l}71 \% \\
98 \%\end{array}$ & $\begin{array}{c}50 \% \\
4 \%\end{array}$ \\
\hline Este estudio Gastelbondo (Colombia-2018) & $\begin{array}{l}\text { PA } \\
\text { PI }\end{array}$ & $\begin{array}{c}107 \\
37\end{array}$ & $\begin{array}{l}92 \\
31\end{array}$ & $\begin{array}{l}85,84 \% \\
83,78 \%\end{array}$ & $\begin{array}{c}15 \\
6\end{array}$ & $\begin{array}{l}14,16 \% \\
16,22 \%\end{array}$ & $\begin{array}{l}74 \% \\
25 \%\end{array}$ & $\begin{array}{l}28 \% \\
71 \%\end{array}$ \\
\hline
\end{tabular}

CLDL: coledocolitiasis; PA: probabilidad alta; NPA: no probabilidad alta; PI: probabilidad intermedia; NR: no reportado; ASGE: Asociación Americana de Endoscopia (American Society for Gastrointestinal Endoscopy). 
pacientes descartados fueron expuestos innecesariamente a este último examen (16).

Las diferencias en el éxito de las CPRE en el presente estudio, así como en los otros mencionados ( $85 \%$ frente al 72 y $75 \%$ ), pueden deberse a que en nuestro trabajo el examen se realizó en el $90 \%$ de los que tenían alta probabilidad y en el $20 \%$ de los que presentaron probabilidad intermedia. Asimismo, en este estudio, la tasa de complicaciones de la CPRE fue del 5,5\% y la pancreatitis por CPRE se constituyó en la más frecuente: se presentó en 6 pacientes $(4,13 \%)$, mientras que la otra complicación fue el sangrado en la papila, la cual ocurrió en 2 pacientes $(1,37 \%)$. Ninguna complicación fue fatal.

La incidencia de complicaciones en este estudio es similar a la informada internacionalmente, en la cual la pancreatitis aguda posterior a la CPRE se presenta en el 3,5\% de los pacientes, mientras que el sangrado relacionado con la esfinterotomía se observó en el 1,3\% $(5,6)$. Con respecto al perfil hepático, la mediana de las aminotransferasas (AST/ ALT) mostró valores más altos al ingreso en los pacientes con CLDL, en comparación con los que no tenían CLDL $(207 \mathrm{mg} / \mathrm{dL}$ y $290 \mathrm{mg} / \mathrm{dL}$ frente a $139 \mathrm{mg} / \mathrm{dLy} 263 \mathrm{mg} / \mathrm{dL}$, respectivamente).

Este hallazgo es similar a lo que encontraron Yurgaky y colaboradores, quienes observaron que, en pacientes con cólico biliar, cuando los niveles iniciales elevados de las aminotransferasas descienden a las 48 y $72 \mathrm{~h}$, la probabilidad de CLDL es $>4$. En el desarrollo de nuestro estudio no fue posible evaluar el comportamiento de las aminotransferasas, debido a que las diferentes entidades promotoras de salud (EPS) no autorizaron repetir dichos exámenes en los pacientes. En este contexto, otros autores también han evidenciado que la elevación de las aminotransferasas es útil para predecir CLDL (20).

En efecto, Nathwani y colaboradores (20) reportaron 18 casos de pacientes con CLDL y cólico biliar, quienes tuvieron una marcada elevación de las aminotransferasas, con niveles pico de AST de $1062 \mathrm{mg} / \mathrm{dL}$ y ALT de $1119 \mathrm{mg} / \mathrm{dL}$, y una posterior caída luego del manejo, la cual sucedió entre los 3 a 14 días. Dicha elevación fue el único hallazgo predictivo de esa patología.

De otro lado, Tozzati y colaboradores (Brasil) (21) y Bourgouin y colaboradores (Francia) (22) han llegado a conclusiones similares con respecto a la elevación inicial de las aminotransferasas. De forma sorprendente, la fosfatasa alcalina mostró valores inferiores en los pacientes con CLDL. No encontramos explicación para este hallazgo, excepto que el $9 \%$ de quienes no presentaron CLDL tenían estenosis de la vía biliar, la cual produce una elevación de la fosfatasa alcalina $(23,24)$. La magnitud de la elevación podría explicar el relativo menor valor de la fosfatasa alcalina en aquellos pacientes con CLDL.
En el presente estudio, la concordancia entre la CRM y la CPRE fue absoluta, ya que cuando la primera visualizó cálculos en el colédoco, estos fueron confirmados por CPRE en el $100 \%$ de los pacientes. En contraste, cuando no se evidenciaron cálculos, la CPRE realizada por alta probabilidad encontró CLDL en el $50 \%$ de los pacientes. Estos resultados difieren con los de publicaciones previas, que han atribuido a la CRM una sensibilidad del $95 \%$, una especificidad del $84,62 \%$, un VPP del 70,37 \% y un VPN del $97,8 \%(25)$.

Así, en nuestro estudio, el VPP fue del $100 \%$, mientras que el VPN fue del $50 \%$. No tenemos una explicación clara para esta disimilitud. Sin embargo, estos datos son semejantes a los encontrados en Colombia por Vargas y colaboradores (11). El VPN del $50 \%$ de la CRM en pacientes con probabilidad intermedia plantea la posibilidad de utilizar preferiblemente la ecoendoscopia biliopancreática, con lo cual las CPRE negativas serían menos frecuentes, tal y como ha sido reportado por otros autores $(26,27)$. No obstante, la elección dependerá de la disponibilidad local y de la calidad del examen, ya que, en gran medida, esto se ve condicionado por el profesional que lo realiza.

La exactitud de las guías de la ASGE en los pacientes con probabilidad alta fue del $67 \%$, valor similar a los encontrados por Suárez y colaboradores (63 \%) (28) y por Ebrahim y colaboradores (65 \%) (19). La exactitud de las guías británicas en este tipo de pacientes fue bastante inferior (39\%).

Entre tanto, el rendimiento de los algoritmos de la ASGE y la BSG para la probabilidad intermedia fue malo, con una sensibilidad apenas cercana al $40 \%$ y una especificidad del $71 \%$. En los pacientes con una alta probabilidad, la sensibilidad de ambas guías es mejor, pero la de la ASGE muestra una leve superioridad ( $74 \%$ frente al $65 \%$ ). La especificidad es muy mala para las dos (ASGE: $28 \%$; BSG: $33 \%$ ).

$\mathrm{Al}$ comparar nuestro trabajo con otros estudios, como se describe en la Tabla 11, los de Sethi y colaboradores (10) y Narváez y colaboradores (17) muestran también una baja sensibilidad en la probabilidad intermedia, a pesar de tener poblaciones muy diferentes. Estos hallazgos refuerzan la necesidad de determinar localmente el mejor examen no invasivo previo a la CPRE.

Así pues, nuestro estudio tiene limitaciones. A pesar de haberse realizado en un hospital universitario, algunas pruebas de laboratorio solicitadas en forma dinámica -y que habrían contribuido al análisis de los pacientes con CLDL- no pudieron realizarse dado que su ejecución necesita la autorización de los auditores de las EPS. Esta circunstancia limita las investigaciones que podrían contribuir al conocimiento y a un mejor manejo futuro de los pacientes. Este actual modelo colombiano de la atención médica restringe la investigación científica del país. 


\section{CONCLUSIONES}

En general, los criterios diagnósticos de la ASGE y la BSG no tienen un buen desempeño en la población estudiada, a fin de discriminar la existencia o no de CLDL. No obstante, en los pacientes con probabilidad alta, estas guías muestran un mejor rendimiento. Con base en nuestros hallazgos, los criterios de la ASGE, aún con sus limitaciones, se deben seguir utilizando y no deben reemplazarse tan prematuramente por los de la BSG. Al considerarse los excelentes resultados de Yurgaky y colaboradores sobre el comportamiento de las aminotransferasas en el tiempo y la elevación inicial de estas (elevación que fue corroborada en este estudio), sería conveniente diseñar un nuevo algoritmo que las incluya en el abordaje de los pacientes con sospecha de CLDL.

\section{Contribución de los autores en la presente investigación}

William Otero, Martín Gómez y Johanna Gastelbondo plantearon la pregunta de investigación y conjuntamente diseñaron el protocolo. JG recolectó la información y, a partir del estadístico, se realizaron los respectivos análisis matemáticos. WO revisó las conclusiones estadísticas. Todos los autores participaron en la discusión de los resultados.

\section{Agradecimientos}

Los autores agradecen el entusiasmo y la colaboración de todos los miembros de nuestra unidad, los doctores Germán Junca, Hernán Ballén, Óscar Ruiz, Javier Riveros, Luis Alberto Ángel y Álvaro Rodríguez, durante todas las fases de este proyecto. Los doctores Martín Gómez, Germán Junca, Óscar Ruiz y Hernán Ballén realizaron las CPRE. Asimismo, agradecemos a todo el personal de Enfermería de la unidad y a los miembros del Departamento de Radiología e Imágenes Diagnósticas de la institución. Un agradecimiento especial a la epidemióloga Diana Buitrago por los análisis estadísticos.

\section{REFERENCIAS}

1. European Association for the Study of the Liver (EASL). Electronic address: easloffice@easloffice.eu. EASL Clinical Practice Guidelines on the prevention, diagnosis and treatment of gallstones. J Hepatol. 2016;65(1):146-181. http://doi.org/10.1016/j.jhep.2016.03.005

2. Csendes A, Diaz JC, Burdiles P, Maluenda F, Morales E. Risk factors and classification of acute suppurative cholangitis. Br J Surg. 1992;79(7):655-8. http://doi.org/10.1002/bjs. 1800790720

3. Yang MH, Chen TH, Wang SE, Tsai YF, Su CH, Wu CW, Lui WY, Shyr YM. Biochemical predictors for absence of common bile duct stones in patients undergoing laparoscopic cholecystectomy. Surg Endosc. 2008;22(7):1620-4. http://doi.org/10.1007/s00464-007-9665-2

4. Costi R, Sarli L, Caruso G, Iusco D, Gobbi S, Violi V, Roncoroni L. Preoperative ultrasonographic assessment of the number and size of gallbladder stones: is it a useful predictor of asymptomatic choledochal lithiasis? J Ultrasound Med. 2002;21(9):971-6.

http://doi.org/10.7863/jum.2002.21.9.971

5. Anderson MA, Fisher L, Jain R, Evans JA, Appalaneni V, Ben Menachem T, Cash BD, Anton G, Early DS, Fanelli RD, Fisher DA, Fukami N, Ha Hwang J, Ikenberry SO, Jue TL, Khan KM, Krinsky ML, Malpas PM, Maple JT, Sharaf RN, Shergill AK, Dominitz JA. Complications of ERCP. Gastrointest Endosc. 2012;75(3):467-473. http://doi.org/10.1016/j.gie.2011.07.010
6. Masci E, Toti G, Mariani A, Curioni S, Lomazzi A, Dinelli M, Minoli G, Crosta C, Comin U, Fertitta A, Prada A, Passoni GR, Testoni PA. Complications of diagnostic and therapeutic ERCP: a prospective multicenter study. Am J Gastroenterol. 2001;96(2):417-23. http://doi.org/10.1111/j.1572-0241.2001.03594.x

7. ASGE Standards of Practice Committee, Maple JT, BenMenachem T, Anderson MA, Appalaneni V, Banerjee S, Cash BD, Fisher L, Harrison ME, Fanelli RD, Fukami N, Ikenberry SO, Jain R, Khan K, Krinsky ML, Strohmeyer $\mathrm{L}$, Dominitz JA. The role of endoscopy in the evaluation of suspected choledocholithiasis. Gastrointest Endosc. 2010;71(1):1-9. http://doi.org/10.1016/j.gie.2009.09.041

8. Williams E, Beckingham I, El Sayed G, Gurusamy K, Sturgess R, Webster G, Young T. Updated guideline on the management of common bile duct stones (CBDS). Gut. 2017;66(5):765-782. http://doi.org/10.1136/gutjnl-2016-312317

9. Adams MA, Hosmer AE, Wamsteker EJ, Anderson MA, Elta GH, Kubiliun NM, Kwon RS, Piraka CR, Scheiman JM, Waljee AK, Hussain HK, Elmunzer BJ. Predicting the likelihood of a persistent bile duct stone in patients with suspected choledocholithiasis: accuracy of existing guidelines and the impact of laboratory trends. Gastrointest Endosc. 2015;82(1):88-93.

http://doi.org/10.1016/j.gie.2014.12.023 
10. Sethi S, Wang F, Korson AS, Krishnan S, Berzin TM, Chuttani R, Pleskow DK, Sawhney MS. Prospective assessment of consensus criteria for evaluation of patients with suspected choledocholithiasis. Dig Endosc. 2016;28(1):75-82. http://doi.org/10.1111/den.12506

11. Vargas RD, Córdoba CP, Uriza LF, Costa V, MosqueraKlinger G, Ortega DA. Concordancia entre los hallazgos por colangiopancreatografía por resonancia magnética y los hallazgos por colangiopancreatografía endoscópica retrograda en pacientes hospitalizados por enfermedad biliar litiásica en el Hospital Universitario San Ignacio (BogotáColombia) entre los años 2005 a 2011. Rev Gastroenterol. 2015;35(3):226-230.

12. Gómez M, Pion J, Otero W. Predictores de coledocolitiasis en pacientes sometidos a colangiografía retrógrada endoscópica en el Hospital El Tunal de Bogotá. Rev Col Gastroenterol. 2011;26(4):243-252.

13. Jurgaky JM. Prevalencia y factores de riesgo de elevación de transaminasas en pacientes con coledocolitiasis. Bogotá: Universidad Nacional de Colombia; 2017.

14. Rubin MI, Thosani NC, Tanikella R, Wolf DS, Fallon MB, Lukens FJ. Endoscopic retrograde cholangiopancreatography for suspected choledocholithiasis: testing the current guidelines. Dig Liver Dis. 2013;45(9):744-9. http://doi.org/10.1016/j.dld.2013.02.005

15. Magalhães J, Rosa B, Cotter J. Endoscopic retrograde cholangiopancreatography for suspected choledocholithiasis: From guidelines to clinical practice. World J Gastrointest Endosc. 2015;7(2):128-34. http://doi.org/10.4253/wjge.v7.i2.128

16. Adams MA, Hosmer AE, Wamsteker EJ, Anderson MA, Elta GH, Kubiliun NM, Kwon RS, Piraka CR, Scheiman JM, Waljee AK, Hussain HK, Elmunzer BJ. Predicting the likelihood of a persistent bile duct stone in patients with suspected choledocholithiasis: accuracy of existing guidelines and the impact of laboratory trends. Gastrointest Endosc. 2015;82(1):88-93. http://doi.org/10.1016/j.gie.2014.12.023

17. Narváez-Rivera RM, González-González JA, MonrealRobles R, García-Compean D, Paz-Delgadillo J, GarzaGalindo AA, Maldonado-Garza HJ. Accuracy of ASGE criteria for the prediction of choledocholithiasis. Rev Esp Enfer. 2016;108(6):309-314. http://doi.org/10.17235/reed.2016.4212/2016

18. Benites HE, Palacios FV, Asencios JL, Aguilas R, Segovia NS. Rendimiento de los criterios predictivos de la ASGE en el diagnóstico de coledocolitiasis en el Hospital Edgardo Rebagliati Martins. Rev Gastroenterol. 2017;37(2):111-119.

19. Ebrahim M, Sorensen LT, Jorgensen LN, Kalaitzakis E. Current clinical algorithms for predicting common bile duct stones have only moderate accuracy. Dig Endosc. 2018;30(4):477-484. http://doi.org/10.1111/den.12994

20. Nathwani RA, Kumar SR, Reynolds TB, Kaplowitz N. Marked elevation in serum transaminases: an atypical presentation of choledocholithiasis. Am J Gastroenterol. 2005;100(2):295-298. http://doi.org/10.1111/j.1572-0241.2005.40793.x

21. Tozatti J, Mello AL, Frazon O. Predictor factors for choledocholithiasis. Arq Bras Cir Dig. 2015;28(2):109-112. http://doi.org/10.1590/S0102-67202015000200006

22. Bourgouin S, Truchet X, Lamblin G, De Roulhac J, Platel JP, Balandraud P. Dynamic analysis of commonly used biochemical parameters to predict common bile duct stones in patients undergoing laparoscopic cholecystectomy. Surg Endosc. 2017;31(11):4725-4734. http://doi.org/10.1007/s00464-017-5549-2

23. Stepien M, Fedirko V, Duarte-Salles T, Ferrari P, Freisling $\mathrm{H}$, Trepo E, Trichopoulou A, Bamia C, Weiderpass E, Olsen A, Tjønneland A, Overvad K, Boutron-Ruault MC, Fagherazzi G, Racine A, Kühn T, Kaaks R, Aleksandrova K, Boeing H, Lagiou P, Benetou V, Trichopoulos D, Palli D, Grioni S, Tumino R, Naccarati A, Panico S, Bueno-deMesquita HB, Peeters PH, Lund E, Quirós JR, Nápoles OC, Sánchez MJ, Dorronsoro M, Huerta JM, Ardanaz E, Ohlsson B, Sjöberg K, Werner M, Nystrom H, Khaw KT, Key TJ, Gunter M, Cross A, Riboli E, Romieu I, Jenab M. Prospective association of liver function biomarkers with development of hepatobiliary cancers. Cancer Epidemiol. 2016;40:179-87. http://doi.org/10.1016/j.canep.2016.01.002

24. Woo YS, Lee KH, Lee KT, Lee JK, Kim JM, Kwon CHD, Joh JW, Kang D, Cho J. Postoperative changes of liver enzymes can distinguish between biliary stricture and graft rejection after living donor liver transplantation: A longitudinal study. Medicine (Baltimore). 2017;96(40):e6892. http://doi.org/10.1097/MD.0000000000006892

25. Perales SR, Souza LRMF, Crema E. comparative evaluation of magnetic resonance cholangiopancreatography and perioperative cholangiography in patients with suspect choledocholithiasis. Arq Bras Cir Dig. 2019;32(1):e1416. http://doi.org/10.1590/0102-672020180001e1416

26. Makmun D, Fauzi A, Shatri H. Sensitivity and Specificity of Magnetic Resonance Cholangiopancreatography versus Endoscopic Ultrasonography against Endoscopic Retrograde Cholangiopancreatography in Diagnosing Choledocholithiasis: The Indonesian Experience. Clin Endosc. 2017;50(5):486-490. http://doi.org/10.5946/ce.2016.159

27. Meeralam Y, Al-Shammari K, Yaghoobi M. Diagnostic accuracy of EUS compared with MRCP in detecting choledocholithiasis: a meta-analysis of diagnostic test accuracy in head-to-head studies. Gastrointest Endosc. 2017;86(6):986-993. http://doi.org/10.1016/j.gie.2017.06.009

28. Suárez AL, LaBarre NT, Cotton PB, Payne KM, Coté GA, Elmunzer BJ. An assessment of existing risk stratification guidelines for the evaluation of patients with suspected choledocholithiasis. Surg Endosc. 2016;30(10):4613-4618. http://doi.org/10.1007/s00464-016-4799-8 